\title{
Combined partial deficiency of muscle carnitine palmitoyltransferase and carnitine with autosomal dominant inheritance
}

\author{
VICTOR IONASESCU*, GEORGE HUG $\dagger$, AND CHARLES HOPPEL \\ University of Iowa Hospitals*, Children's Hospital Research Foundation Cincinatti $\dagger$ and the Veterans \\ Administration Hospital Cleveland $\ddagger$
}

SUMMARY The authors studied a 53 year old woman and her 22 year old son with episodes of paroxysmal muscle cramps and dark urines lasting several hours related to high fat diet and strenuous physical exercise beginning on both at age 14 years. The father, paternal uncle, paternal grandfather and another son of the mother also had paroxysmal muscle cramps. The two studied cases showed normal findings for physical evaluation, blood lactate after ischemic exercise, and muscle histology (light and electron microscopy). The serum creatine kinase was elevated in the son and normal in the mother. However, 72 hour fasting significantly raised the serum creatine kinase level in both cases. Plasma concentration of ketone bodies and acid soluble acyl-carnitine increased normally with prolonged fasting. The biochemical evaluation of the muscle tissue revealed intact anaerobic glycolysis and normal glycogen content but combined partial deficiency of muscle carnitine palmitoyltransferase and carnitine in both cases.

Severe paroxysmal muscle cramps usually represent a feature of defective glycogen muscle metabolism such as deficiency of muscle phosphorylase and phosphofructokinase. They may be accompanied by myoglobinuria. Myoglobinuria and atypical muscle complaints have also been reported in cases with deficiency of carnitine palmitoyltransferase (CPT) in skeletal muscle. ${ }^{1-3}$ We report below two related patients with paroxysmal muscle cramps and partial deficiency of both muscle CPT and carnitine. This disease is inherited as an autosomal dominant trait in four generations.

\section{CASE REPORT}

Case 1 A 53 year old woman experienced episodes of muscle cramps since she was 14 years old. These occurred every three to four weeks, manifested as moderate aching in the muscles of the legs, arms or both. The muscle cramps were related to strenuous physical activity such as long walks, climbing in the mountains or dancing for many hours. One of

Address for reprint requests: Dr Victor Ionasescu, Department of Pediatrics, University of Iowa Hospitals, Iowa City, IA 52242, USA.

Accepted 7 February 1980 the worst episodes occurred when she was 25 years old during a dance competition, where she danced for the whole night. The muscle cramps were more frequent and severe during her five pregnancies. High fat diet could also induce these muscle cramps but with milder intensity. There was no weakness accompanying the muscle cramps. Several episodes of paroxysmal pains were followed by the passage of dark brown urines lasting for one to two days. Her family history shows that her father, a paternal uncle, paternal grandfather and two of her sons (fig 1) also experienced paroxysmal muscle cramps and dark urines. Her father and paternal uncle also had a liver condition which was diagnosed as hemochromatosis on the basis of a liver biopsy. The frequency and intensity of her episodes of muscle cramps had significantly decreased for the last three years when the patient avoided strenuous physical activity and had a low fat diet.

Her past medical history was noncontributory. She was a slightly overweight patient with no neurological abnormality and had good muscle strength.

Case 2 A 22 year old patient, son of Case 1, had the clinical onset of his condition at 


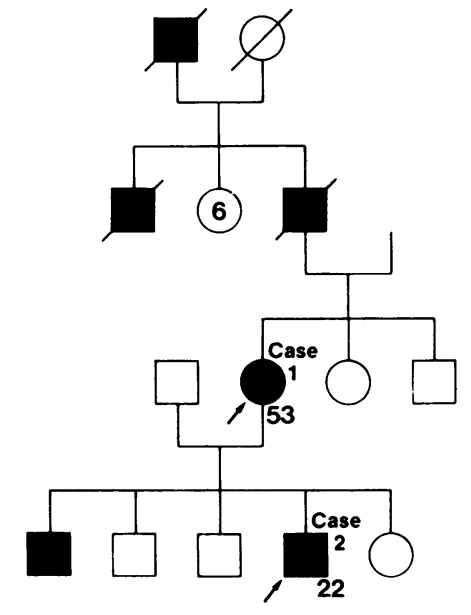

Fig 1 Pedigree showing autosomal dominant inheritance of the condition with patients in four generations.

14 years of age like his mother. He noticed severe muscle cramps and weakness in his legs during wrestling in contrast to earlier years when he was able to wrestle with several consecutive partners in a row without getting tired. On several occasions he has voided dark coloured urine. Initial frequency of the paroxysmal muscle cramps was one to two per week. There was a significant drop in the number and regularity of these episodes after the patient discontinued wrestling. The duration of muscle cramps has also decreased from 24 hours to 30 minutes, without accompanying weakness or dark urine. The high fat diet as in his mother could also induce cramps in his extremities.

Past medical history was noncontributory. Examination showed a normal male of an athletic build.

\section{Methods}

Muscle samples from left vastus lateralis were frozen in liquid nitrogen immediately after biopsy and stored in a liquid nitrogen container until used. Carnitine and carnitine palmitoyltransferase were measured according to the method of Pearson et al, ${ }^{4}$ Crabtree et al. ${ }^{5}$ Glycogen and phosphorylase were measured by Hug procedure. ${ }^{6}$ Debrancher enzyme was measured by the method of Hers. ${ }^{7}$

Metabolic studies with fasting: The patients were fasted for 72 hours and blood samples were obtained three times daily for the determination of carnitine by the procedure of Brass and Hoppel, ${ }^{8}$ plasma free fatty acids by the procedure of Dole, 9 beta hydroxybutyrate and acetoacetate by the procedure of Cahill et $a l^{10}$ and creatine kinase (CK) by the procedure of Szasz. ${ }^{11}$ Control subjects matched for age and sex were fasted for the same period of time and with the same methods by Hoppel (unpublished observations).

Muscle histology: A portion of the muscle biopsy was fixed for three hours in 3\% glutaraldehyde in $0.1 \mathrm{M}$ phosphate buffer at $\mathrm{pH} 7 \cdot 4$ for electron microscopy. Tissue blocks were stained with $2 \%$ uranyl acetate in $10 \%$ ethanol before dehydration and embedding. Standard techniques were used for the preparation of thick and thin sections. Sections were stained with uranyl acetate and lead citrate and examined in a Siemens 101 electron microscope. Tissue was also processed for paraffin sections and histochemical study of frozen sections.

\section{Results}

Table 1 shows a partial deficiency of muscle CPT in both cases. The enzyme was decreased by $39 \%$ in Case 1 and $40 \%$ in Case 2. A partial deficiency is also present for muscle carnitine which was decreased by $59 \%$ in Case 1 and by $67 \%$ in Case 2. In contrast glycogen concentration and glycolytic compounds showed normal values in both cases. There is good agreement between these data and the forearem ischaemic exercise

Table 1 Muscle biochemical findings

\begin{tabular}{llll}
\hline & Control & Case 1 & Case 2 \\
\hline $\begin{array}{l}\text { Carnitine palmitoyltransferase } \\
\text { (nmoles/g wet weight) }\end{array}$ & $387.7 \pm 69.96$ & 249 & 234 \\
$\begin{array}{l}\text { Carnitine } \\
\text { (nmoles/g wet weight) }\end{array}$ & $3,890 \pm 1.170$ & 1,610 & 1,270 \\
$\begin{array}{l}\text { Glycogen concentration } \\
\text { (percent/wet weight) }\end{array}$ & $<1.5 \%$ & 0.63 & 0.53 \\
$\begin{array}{l}\text { Phosphorylase } \\
\text { ( } \mu \text { moleP/min/g tissue) }\end{array}$ & $47.7 \pm 13 \cdot 2$ & 59.0 & 80.7 \\
$\begin{array}{l}\text { Debrancher enzyme } \\
\text { (cpm/mcg glycogen/g/hr) }\end{array}$ & 2.490 & 3.920 & 4,957 \\
\hline
\end{tabular}

Table 2 Serum and plasma biochemical findings

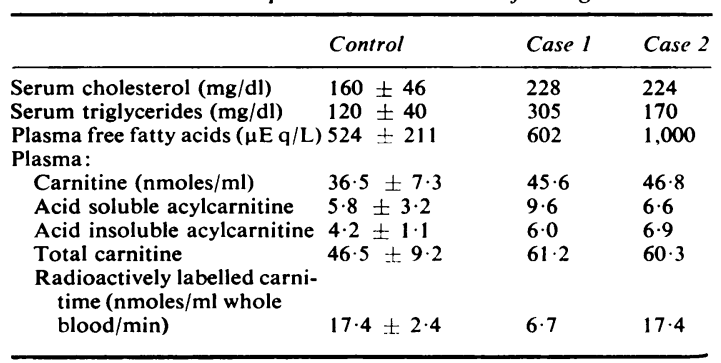




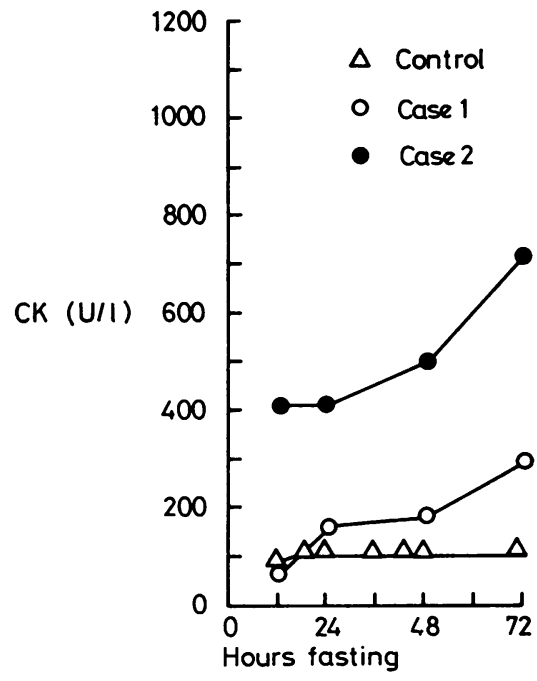

Fig 2 Effect of prolonged fasting on the serum creatine kinase.

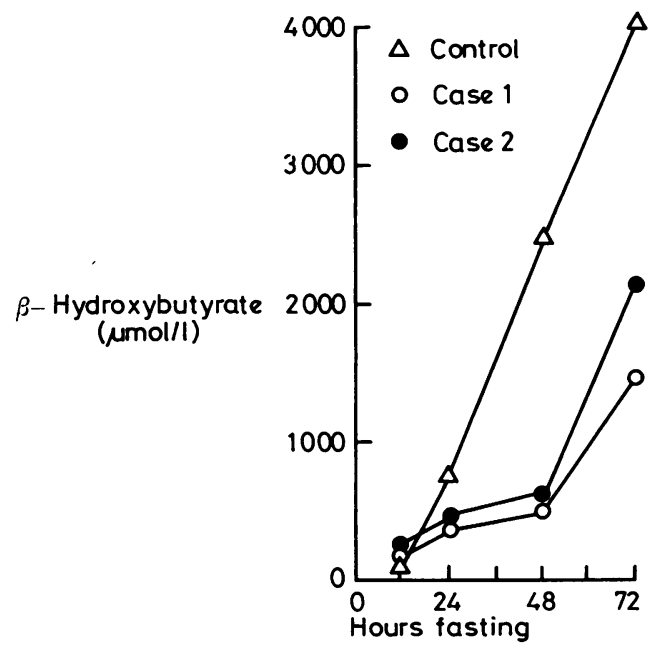

Fig 3 Effect of prolonged fasting on beta-hydroxybutyrate. which showed normal increases of lactate and pyruvate for both cases.

Table 2 shows decreased radioactively labelled carnitine in Case 1, but normal values in Case 2. Case 1 showed a $250 \%$ increase in serum triglycerides while Case 2 showed values at the upper normal limit. Both cases showed normal values for serum cholesterol. Plasma free fatty acids are within normal limits for Case 1 but 90\% elevation for Case 2. Serum CK showed normal values in Case 1, but significantly higher values in Case 2. The fasting prompted an abnormal increase in serum CK in both cases (fig 2). The fasting showed a normal increase in beta hydroxybutyrate (fig 3 ), acetoacetate and free fatty acids.

The muscle histology (light and electron microscopy) showed normal muscle structure without any accumulation of lipids. The liver tests (SGOT, SGPT) showed normal values. The evaluation of the urine could not detect myoglobin in a cramp free period in both patients.

\section{Discussion}

Partial deficiency of muscle CPT was first described by Hostetler et $\mathrm{al}^{12}$ in a 28 year old patient with recurrent myoglobinuria and atypical muscle complaints. The enzyme in their case was decreased by $54 \%$. The decrease in enzymatic activity in our cases was $39 \%$ for Case 1 and $40 \%$ for Case 2. Partial deficiency of muscle carnitine has not yet been reported in undamaged muscle. The decrease reached $59 \%$ in Case 1 and $67 \%$ in Case 2. Case 1 also showed a significantly low level of radioactively labelled carnitine in whole blood (table 2). The unusual combination of partial deficiency of muscle CPT and carnitine might represent a new disease with different inheritance from CPT and carnitine deficiency. It is interesting that although the biochemical findings show a more severe deficiency for carnitine than for CPT, the clinical findings fit more the picture of CPT deficiency. Both patients show paroxysmal muscle cramps, accompanied by dark brown urine suggesting myoglobinuria although we could not confirm this. In Case 2, muscle weakness occurs also at the time of muscle cramps. Both patients show muscle cramps in conditions of strenuous physical exercise like the other cases of CPT deficiency. ${ }^{1-3}$ In addition, they show muscle cramps with high fat diet which are controlled by an adequate change in their food intake. The physical 
evaluation between the episodes of muscle cramps showed normal findings in both our patients with no evidence of myopathy. The light and electron microscopy did not reveal any alterations of muscle structure or accumulation of lipids which again resembles most of the cases with CPT deficiency. The biochemical evaluation of the muscle tissue revealed intact anaerobic glycolysis and normal glycogen content (table 1).

Seventy-two hours fasting significantly raised the serum CK level (fig 2) in both cases as previously reported in CPT deficiency by Hostetler et $a l^{12}$ and Carrol et al. $^{3}$ Plasma concentration of ketone bodies (fig 3 ) and acid soluble acyl-carnitine increased normally with prolonged fasting in contrast to the cases described by Bank et $a l^{13}$ where ketone production was considerably reduced suggesting a decreased level of CPT in the liver in addition to its low muscle level.

All cases reported with carnitine or CPT deficiency were either sporadic or showed definite autosomal recessive inheritance. ${ }^{14}$ These are the first reported cases with combined CPT and carnitine deficiency which show autosomal dominant inheritance supporting the concept of a different disease. The association of paroxysmal muscle cramps and haemochromatosis in two previous cases from the same family is interesting. However, the role of haemochromatosis in the pathogenesis of this disease seems to be limited since our patients do not show signs of haemochromatosis and their liver functional tests were normal.

This work was supported by United States National Institutes of Health Grants RR 05535-17 and RR 00123.

\section{References}

1 Di Mauro S, Melis-Di Mauro PM. Muscle carni- tine palmitoyltransferase deficiency and myoglobinuria. Science 1973; 182:929-30.

2 Angelini C. Lipid storage myopathies: a review of metabolic defects and of treatment. J Neurol 1976; 24:1-11.

3 Carroll JE, Brooke MN, DeVivo DC, Kaiser KK, and Hagberg JM. Biochemical and physiologic consequences of carnitine palmitoyltransferase deficiency. Muscle and Nerve 1978; 1:103-10.

4 Pearson DJ, Tubbs PK. Carnitine and derivatives in rat tissues. Biochem $J$ 1967; 105:953-63.

5 Crabtree B, Newsholine EA. The activities of lipases and carnitine palmitoyltransferase in muscles from vertebrates and invertebrates. Biochem J 1972; 130:697-705.

6 Hug G, Schubert WK, Chuck G. Deficient activity of dephosphorylase-kinase and accumulation of glycogen in the liver. J Clin Invest 1969 ; 48:704-15.

7 Hers HG. Etudes enzymatiques sur fragments hepatiques: application a la classification des glycogenoses. Rev Int Hepatol 1959; 9:35-55.

8 Brass EP, Hoppel CL. Carnitine metabolism in the fasting rat. J Biol Chem 1978; 253:2688-93.

9 Dole VP. A relation between non-esterified fatty acids in plasma and the metabolism of glucose. J Clin Invest 1956; 35:150-4.

10 Cahill GF Jr, Herrera MG, Morgan AP et al. Hormone-fuel interrelationships during fasting. $J$ Clin Invest 1966; 45:1751-69.

11 Szasz G, Gruber W, Bernt E. Creatine kinase in serum: 1. Determination of optimum reaction conditions. Clin Chem 1976: 22:650-6.

12 Hostetler KY, Hoppel CL, Romine JS, Sipe JC, Gross SR, Higginbottom PA. Partial deficiency of carnitine palmitoyltransferase with normal ketone production. $N$ Engl J Met 1978; 298: 553-7.

13 Bank WJ, di Mauro S, Bonilla E. Capuzzi DM, Rowland LP. A disorder of muscle lipid metabolism and myoglobinuria: absence of carnitine palmitoyltransferase. $N$ Engl J Med 1975; 292: 443-9.

14 Reza MJ, Kar NC, Pearson CM, Pieterkark RA. Recurrent myoglobinuria due to muscle carnitine palmitoyltransferase deficiency. Ann Int Med 1978; 88:610-5. 